\title{
Surface Localization in Impurity Band with Random Displacements and Long-Range Interactions
}

\author{
Victor Chulaevsky (D) \\ Département de Mathématiques, Université de Reims, Moulin de la Housse, BP 1039, 51687 Reims Cedex 2, France \\ Correspondence should be addressed to Victor Chulaevsky; plinius@free.fr
}

Received 28 September 2017; Revised 22 December 2017; Accepted 4 January 2018; Published 8 April 2018

Academic Editor: Hagen Neidhardt

Copyright ( 2018 Victor Chulaevsky. This is an open access article distributed under the Creative Commons Attribution License, which permits unrestricted use, distribution, and reproduction in any medium, provided the original work is properly cited.

\begin{abstract}
We study the random Schrödinger operators in a Euclidean space with the disorder generated by two complementary mechanisms: random substitution in a lower-dimensional layer and random displacements in the bulk, without additional assumptions regarding the reflection symmetry of the site potentials. The latter are assumed to be bounded and have a power-law decay. Complementing earlier results obtained in the strong disorder regime, we establish spectral and strong dynamical localization in the impurity zone near the bottom of spectrum for arbitrarily weak amplitudes of the random displacements, provided the concentration of impurities is sufficiently small.
\end{abstract}

\section{Introduction}

1.1. The Model. By a random displacements Hamiltonian in $\mathbb{R}^{d}$ one usually calls a random Schrödinger operator of the form

$$
H(\omega)=-h \Delta+V_{0}(x)+\sum_{a \in \mathscr{X}^{d}} \mathfrak{t}_{a}\left(x-X_{a}(\omega)\right),
$$

where $h>0, \mathscr{Z}^{d} \subset \mathbb{R}^{d}$ is a discrete lattice (usually periodic), and $X_{a}(\omega)$ are random positions of the "scatterers" labeled by the lattice sites $a$ and characterized by the local potentials ("scatterer potentials") $\mathfrak{t}_{a}$. In particular, all $\mathfrak{t}_{a}$ may be identical. Here $V_{0}(x)$ is a nonrandom component of the potential generated by the media, which one may choose to be trivial $\left(V_{0}(x) \equiv 0\right)$ or not. Generally speaking, such models are more difficult to study than the random alloy models with Hamiltonians

$$
-h \Delta+\sum_{a \in \mathscr{X}} c_{a}(\omega) \mathfrak{t}_{a}(x-a)
$$

where the randomness resides in the IID (independent and identically distributed) amplitudes $c_{a}(\omega)$, and the latter are usually assumed to have a Lipschitz- or Hölder-continuous probability distributions.
There is a considerable wealth of mathematical research papers and monographs on alloy models and their finitedifference analogs, while the number of rigorous results on the random displacements models (RDM, in short) remains quite limited.

Both in the class of alloy models and the RDM, one usually assumes $\mathfrak{i t}$ to be compactly supported; this is of course a conscious simplification of the physical reality where the fundamental interactions have infinite range. Indeed, a rigorous study of long-range models is substantially more complicated due to the long-range correlations induced by $\mathfrak{u}$.

In the present paper, we treat a model which combines these two features: the randomness is provided only by the displacements $X_{a}(\omega)$ and the potentials $\mathfrak{u}$ decay polynomially at infinity. To be more precise, we also introduce an additional disorder mechanism, random substitutions of scatterer potentials, as described below.

Klopp [1] studied the random displacements model with the help of powerful techniques of the semiclassical analysis, hence under the assumption that the kinetic energy amplitude $h$ in $-h \Delta$ is sufficiently small. Since multiplication of an operator by a nonzero constant does not change the nature of its spectral components (absolutely continuous, singular continuous, and pure point spectra), the assumption $h \ll 1$ in $H=-h \Delta+V(\omega)$ is equivalent to the assumption $h^{-1} \gg 1$ 
in the rescaled operator $h^{-1} H(\omega)=-\Delta+h^{-1} V(\omega)$; the latter is usually called the strong disorder regime. The semiclassical approach has a number of important advantages; in particular, it resulted in [1] in the proof of a Wegner-type estimate and of the Initial Length Scale (ILS) estimate based on the Lifshitz tails phenomenon (cf. [2-4]). However, an adaptation of these technically involved methods to the random displacements models with an infinite-range, slowly decaying interaction potential $\mathfrak{u}$ is far from being straightforward, and it is beyond the scope of the present paper.

Lott and Stolz [5] and Baker et al. [6, 7] studied the spectral minimizers for the displacements model, in the context, and obtained important analytic (deterministic) results in this direction. Further progress was obtained by Klopp et al. [8] who described the energy minimizing configurations in the displacements model, assuming that the site potential is reflection-symmetric in each coordinate variable $x_{i}=0$, $1 \leq i \leq d$ (see the precise assumptions (A1) and (A2) in [8]), and proved Anderson localization in their model for energies near the bottom of the spectrum.

We consider the model where the local potentials are of two different types. Physically, it corresponds to a disordered media sample with different types of atoms (or ions, when some electrons are mobile; for simplicity we will always speak of "atoms" bearing the respective local potentials). Generally speaking, one can consider the models with an arbitrary number $K>1$ of atoms, but the most important feature of our model is that in some sublattice $\mathscr{Z}^{d_{0}} \hookrightarrow \mathscr{Z}^{d}=\mathscr{Z}^{d_{0}} \times \mathscr{Z}^{d_{1}}$ some randomly selected atoms are of one particular type with potentials taking values below those of all other types (negative, in our model).

A realistic example is given by a thin film (some recent CPU chips are built on $14 \mathrm{~nm}$ films, with just a few atoms across it) where one thin filament is "doped" by randomly placed atoms producing a low energy band: below the main energy band of the bulk material. Similarly, in 3 dimensions, one can think of a surface of a crystal with randomly places impurities; such a layer, producing a low energy band, can be sandwiched between two much thicker samples of the bulk material. A thorough examination of our arguments in the subsequent sections evidences that the main results can be easily extended to a model where the impurities are randomly placed inside a finite-width layer and not just a monolayer formed by a sublattice $\mathscr{Z}^{d_{0}}$ of nonzero codimension. Our technique allows one to prove exponential spectral and strong dynamical localization in the impurity zone produced solely by randomness in local displacements within a subsample of the bulk material (located at a finite distance from the impurity layer).

From the point of view of experimental physics and new technologies, an approach relying only on random displacements provides a solid base for the emergence of Anderson localization, where more traditional random alloy models rely crucially on the fluctuations of the strength of the local potentials $\omega_{a} \mathfrak{t}(x-a)$, with $\omega_{a}$ having a continuous (even absolutely continuous) probability distribution. It is quite clear from the basic facts of the electrostatic interactions that the amplitude of the potential is proportional to the electric charge of a given ion, and the latter is an integer multiple of the electron charge $e$, so it cannot have a continuous distribution. Of course, the popular alloy models implicitly refer to a complex structure of the ionic potentials, yet the question of whether or not the latter could result in a nice probability distribution of local potential amplitudes is far from obvious, and this is where the random displacements mechanism proves much more pertinent and universal.

To formalize the above considerations, we introduce the extended samples $\boldsymbol{\omega}=(\omega, \vartheta)$ on a probability space $(\Omega, \mathfrak{B}, \mathbb{P})$, where $\omega=\left(\omega_{a}\right)_{a \in \mathscr{T}}$ and $\vartheta=\left(\vartheta_{a}\right)_{a \in \mathscr{E}^{d_{0}}}$ are mutually independent random fields on the entire lattice $\mathscr{Z}$ and on the sublattice $\mathscr{Z}^{d_{0}}$, respectively, and each of them is IID (has independent and identically distributed random values). To make explicit some geometrical constraints we introduce for the sake of clarity, we assume that the intersite distances in $\mathscr{Z}^{d}$ are greater than or equal to 1 (e.g., $\mathscr{Z}^{d}=$ $\mathbb{Z}^{d}$ ), but in the case of more general lattices it suffices to adapt intermediate geometrical parameters. Apart from the difference in the index sets of $\omega_{a}$ and $\vartheta_{a}\left(\mathscr{Z}\right.$ versus $\left.\mathscr{Z}^{d_{0}}\right)$, the respective common marginal probability distributions are quite different:

(U1) $\vartheta_{a}, a \in \mathscr{Z}^{d_{0}}$, are Bernoulli random variables with $\mathbb{P}\left\{\vartheta_{a}=1\right\}=p=1-\mathbb{P}\left\{\vartheta_{a}=0\right\} \in(0,1)$.

(U2) $\omega_{a} \in \mathbb{R}^{d}, a \in \mathscr{Z}$, have a bounded, compactly supported probability density $\rho_{0}(x), x \in \mathbb{R}^{d}$. Specifically, we assume for the sake of clarity of presentation that there exist $0<r_{1}<r_{2}<1 / 2$ such that

(U2.1) supp $\rho_{0} \subset B_{r_{2}}(0)$,

(U2.2) $\rho_{0}(x) \geq c \mathbf{1}_{B_{r_{1}}(0)}(x), c>0$.

Given the random displacements $\omega_{a}$, we denote by $X_{a}:=$ $a+\omega_{a}$ the random positions of the (centers of) the atoms forming the media.

Next, we consider two types of the local potentials, $\mathfrak{t}_{0}$ and $\mathfrak{t}_{1}$. Simply put, the potential $V$ induced by any sample without "impurities" (potentials $\mathfrak{t}_{1}$ ) is nonnegative, while an impurity at $a \in \mathscr{Z}^{d_{0}}$ (implemented by a site potential $\mathfrak{u}_{0}(\cdot-a)$ ) produces a negative potential well in some ball $B_{r_{0}}(a)$, and we assume that this negative well cannot be destroyed by any configuration of atom types and displacements occurring outside some neighborhood of the site $a$.

Specifically, we assume that $\mathfrak{u}_{0}$ and $\mathfrak{u}_{1}$ fulfill the following conditions:

(U3) For $r \geq 1 / 2$ and some $A>2 d$,

(U3.1) $\left|\mathfrak{u}_{1}(r)\right| \leq C_{1} r^{-A}, C_{1} \in(0,+\infty) ;$

(U3.2) $\mathfrak{t}_{0}(r)=r^{-A}$.

From $\mathfrak{u}_{1}(r)$ with $r \geq 1 / 2$ we only need an upper bound (U3.1) on its decay rate, while more detailed analytical information is required for $\mathfrak{u}_{0}(r)$ and its gradient, which explains an explicit choice made in (U3.2).

The main distinction between $\mathfrak{t}_{0}$ and $\mathfrak{t}_{1}$ is explained by the following hypothesis (recall $X_{a}=a+\omega_{a}$ ): 
(U4) There exist some $E^{(-)}<0$ and $r_{0}>0$ such that for and all $x \in B_{r_{0}}(0)$ one has

$$
\mathfrak{t}_{1}(x)+\sum_{a \neq 0} \sup _{\left|\omega_{a}\right| \leq r_{2}} \max _{k=0,1} \mathfrak{i t}_{k}\left(x-X_{a}\right) \leq E^{(-)}
$$

while $\mathfrak{t}_{0}(x) \geq 0$ for all $x \in \mathbb{R}^{d}$.

In addition, we assume that the kinetic energy amplitude $h$ (cf. (1)) is sufficiently small, depending upon $\mathfrak{u}_{0}$, so that the negative wells (starting from a single one) produce deterministically a nontrivial negative spectrum of $H$.

Since (U3.1) gives a deterministic bound on the decay $|\mathfrak{a t}|$ (in fact, it could be assumed in addition to the condition of nonpositivity everywhere), and $\mathfrak{t}_{0}(r)=r^{-A}$ outside a ball of radius $1 / 2$, it suffices to take $\mathfrak{t}_{1}(r)$ negative and sufficiently large in $B_{r_{0}}(0)$ to create a deep negative well. Further, the variational estimates show that such a well around an impurity produces negative spectrum, at least for the amplitude $h$ of the Laplacian small enough.

The main point of the present work is the analysis of the spectral band produced by the $\mathfrak{u}_{0}$-wells.

Introduce the alloy potential in $\mathbb{R}^{d}$ of the form

$$
V(x ; \boldsymbol{\omega})=V^{(B)}(x ; \omega)+V^{(S)}(x ; \omega)
$$

(here $(B)$ stand for "Bulk" and (S) for "Surface"), with

$$
\begin{aligned}
V^{(B)}(x ; \omega)= & \sum_{a \in \mathscr{Z}^{d}} \mathfrak{u}_{0}\left(x-X_{a}\right), \\
V^{(S)}(x ; \omega, \vartheta)= & \sum_{a \in \mathscr{X}^{d_{0}}: \vartheta_{a}=0} \mathfrak{t}_{0}\left(x-X_{a}\right) \\
& +\sum_{a \in \mathscr{E}^{d_{0}}: \vartheta_{a}=1} \mathfrak{t}_{1}\left(x-X_{a}\right)
\end{aligned}
$$

(recall $\left.X_{a}=a+\omega_{a}\right)$.

Once the selection of the potential types $\vartheta_{a} \in\{0,1\}$ is made, the only randomness in the potential sample $V(x ; \boldsymbol{\omega})$ is the one coming from the displacements $X_{a}(\omega)$.

The assumptions on smallness of the support of the probability measure $\rho_{0}$ (of the displacements $\omega_{a}$ ) had been made before in several works on the random displacements models (RDM) where it was actually crucial that the supports of the local potentials, even after admissible displacements of their centers within neighborhoods of the lattice sites $a$, do not overlap, for otherwise the subtle functional-analytic results used in earlier proofs would break down. It will be clear from our presentation that we can afford supp $\rho_{0} \subset$ $B_{\bar{r}}(0)$ with any fixed $\bar{r}<+\infty$, at the price of a more elaborate analysis and some additional assumptions on the site potentials (either a "hard core" condition or smallness of the positive potential $\mathfrak{t}_{0}$, in order not to destroy the impurity wells). This follows from our strategy of proof of the crucial eigenvalue concentration (or Wegner-type) estimate which relies only on the randomness of $\omega_{a}$ with $a$ filling an affine sublattice parallel to $\mathscr{Z}^{d_{0}}$ at some finite distance $r_{2}$ from the latter. The reader will see that the displacements of all remaining random positions $X_{a}=a+\omega_{a}$ can be (and often will be) rendered nonrandom by conditioning. However, this also makes more cumbersome some important geometrical arguments, and for this reason we postpone possible generalizations to a forthcoming work.

Kirsch et al. [9] adapted the variable-energy (or energyinterval) MSA approach developed by Fröhlich et al. [10] (cf. also [11]), reformulated by von Dreifus and Klein [12], to the alloy models with a polynomial decay of the interaction potential $\mathfrak{t}(r)=r^{-A}$, under the assumption $A>4 d$. However, their techniques apply directly to the models featuring the so-called monotonicity condition: a variation of any random parameters (scatterer amplitudes, in their case) has to produce a variation of the potential with fixed sign (either positive or negative), while the displacements of the scatterers result in sign-indefinite variations of the induced potential.

An important issue one has to face in the class of the random displacements models is the proof of the ILS estimate for an arbitrarily small disorder amplitude, without the strong disorder condition. The main point of the present paper is that this issue can be addressed in a relatively simple way for the surface models where a layer of the sample producing a spectral band, separated by a gap from the bulk spectrum, is subject both to the random displacements disorder and to the substitution disorder (due to a random selection of the lattice positions for the impurities).

The latter kind of disorder is "discrete" and not generated by variation of continuous parameters. Such a situation usually renders the localization analysis very difficult; the first result in this area was obtained in a remarkable work by Bourgain and Kenig [13] in 2001, for the random alloys with Bernoulli distribution of random scatterers amplitudes. Germinet and Klein [14] have later extended the Bourgain-Kenig approach to arbitrary nontrivial distributions, but the technique here remains very complex and the localization bounds (for the eigenfunction correlators) remain quite modest. In our paper, we use a "discrete" substitution disorder solely for the proof of the Initial Length Scale estimate, which is already quite difficult to obtain otherwise. To the author's best knowledge, there is no prior result for the weak-disorder localization at low energy in the random displacements model with slowly decaying interaction.

Moreover, we show that the moderate rate of decay of the Green functions obtained at the first stage of the multiscale analysis can be significantly enhanced by a "soft" argument, so that despite a relatively slow, power-law decay of interaction (hence, a relatively strong long-distance correlation in the random environment) the decay of eigenfunction correlators is at least fractional-exponential.

The materials with an impurity band supported by specific atoms injected into a sample play important role in experimental physics and technology. In our paper, we thus show that even a relatively weak disorder induced by structural factors (substitution and local displacements of the atoms/ions) can produce strong dynamical localization in presence of a realistic, slow decay of the media-electron interaction at large distances. 
1.2. Local Hamiltonians and Useful Notation. Given a cube $B_{3 \ell}(u)$, with $\ell \geq 1$, we call $B_{\ell}(u)$ the core and the annulus $B_{L_{k}}(u) \backslash B_{\ell_{k}}(u)$ the shell of $B_{3 \ell}(u)$. Further, given a cube $B_{Y L}(u)$ with $L=3 \ell, Y \geq 2$, we can cover the shell of $B_{Y L}(u)$ by $(3 Y)^{d}$ cores of radius $\ell_{k}$. The respective cores $B_{\ell_{k}}(x)$ will be called admissible.

We define the "boundary" of a cube $B_{L_{k}}(u)$ as follows: $\partial B_{L_{k}}(u):=B_{L_{k}}(u) \backslash B_{L_{k}-2}(u)$.

The spectrum of an operator $H$ will be denoted by $\Sigma(H)$ and its resolvent set by $\varrho(H)$.

\subsection{Main Results}

Theorem 1. Assume that the conditions (U1)-(U4) are fulfilled. Then there exist $\widehat{p}>0$ and an interval $\widehat{I} \subset \mathbb{R}$ such that the following holds for the random displacements model where $p \equiv \mathbb{P}\left\{\vartheta_{a}=1\right\} \in(0, \widehat{p})$.

(A) With probability one, $H(\omega)$ has pure point spectrum in the interval $\widehat{I}$, and all the eigenfunctions $\psi$ of $H(\omega)$ with eigenvalues $E \in \widehat{I}$ decay exponentially at infinity: for some $m>0$ one has

$$
\left|\psi_{x}(y ; \omega ; \vartheta)\right| \leq C_{\psi}(\omega, \vartheta) e^{-m|y-x|} \quad \forall y \in \mathscr{Z} .
$$

(B) Let $\chi_{x}, x \in \mathbb{R}^{d}$, be the indicator function of a unit cube centered at $x$. There exist $C, \varrho \in(0,+\infty)$ such that for all $x \neq y$ and for any bounded Borel function $\phi_{\widehat{I}}$ with $\operatorname{supp} \phi_{\widehat{I}} \subset \widehat{I}$ and $\left\|\phi_{\bar{I}}\right\|_{\infty} \leq 1$ one has

$$
\mathbb{E}\left[\left\|\chi_{x} \phi_{\bar{I}}(H(\omega)) \chi_{y}\right\|\right] \leq C e^{-|x-y|^{e}} .
$$

The smallness of the concentration of impurities $p$ in the "surface" layer is used only in the proof of the Initial Length Scale estimate (cf. Lemma 19).

\section{Eigenvalue Concentration and Comparison Estimates}

2.1. Stollmann's Estimate. Recall some well-known notions and results from the eigenvalue concentration analysis of random operators introduced and used in earlier works [4, 15-18].

Definition 2. Let a finite nonempty index set $J$, a Euclidean space $\mathbb{R}^{J} \cong \mathbb{R}^{|J|}$ with coordinates indexed by the elements of $J$, and a probability measure $\mu$ on $\mathbb{R}$ be given. Introduce the product measure $\mu^{\otimes J}$ on $\mathbb{R}^{J}$. A function $\Phi: \mathbb{R}^{J} \rightarrow \mathbb{R}$ is called diagonally monotone iff

(i) $q=\left(q_{1}, \ldots, q_{J}\right) \mapsto \Phi(q)$ is monotone in each variable $x_{j}, 1 \leq j \leq|J|$,

(ii) for all $q \in \mathbb{R}^{J}$ and $t \geq 0$ one has

$$
\Phi(x+(t, \ldots, t))-\Phi(x) \geq C t .
$$

From this point on, speaking of a diagonally monotone function $\Phi$, we always assume that the respective index $J$,
Euclidean space $\mathbb{R}^{J}$, and a constant $C>0$ are given. To indicate an explicit value of $C$, we sometimes say that $\Phi$ is diagonally $C$-monotone.

Lemma 3 (Stollmann's lemma). Let $\Phi: \mathbb{R}^{J} \rightarrow \mathbb{R}$ be a diagonally C-monotone function and $\mu^{\otimes J}$ a product measure on $\mathbb{R}^{J}$. Let $\mathfrak{S}_{\mu}:[0,+\infty) \rightarrow[0,1]$ be the continuity modulus of $\mu$ (otherwise called the Levy concentration function of $\mu$ ):

$$
\mathfrak{S}_{\mu}: s \longmapsto \sup _{a \in \mathbb{R}} \mu([a, a+s]) \text {. }
$$

Then the following estimate holds true:

$$
\sup _{a \in \mathbb{R}} \mathbb{P}\left\{q \in \mathbb{R}^{J}: \Phi(q) \in[a, a+s]\right\} \leq C^{-1}|J| \mathbb{S}_{\mu}(s) .
$$

Definition 4. Let a Hilbert space $\mathscr{H}$, a Euclidean space $\mathbb{R}^{J}$, $J \geq 1$, and a probability measure $\mu$ on $\mathbb{R}$ be given. A family of self-adjoint operators $H(q)$ in $\mathscr{H}, q \in \mathbb{R}^{J}$, is called diagonally monotone iff

$$
H(q+r) \geq B(q) \quad \forall q \in \mathbb{R}^{J} \forall r \in \mathbb{R}_{+}^{J},
$$

in the sense of quadratic forms, and for any vector $f \in \mathscr{H}$ with $\|f\|=1$ the function $\Phi_{f}: \mathbb{R}^{J} \rightarrow \mathbb{R}$ defined by

$$
\Phi_{f}(q)=(H(q) f, f)
$$

is diagonally monotone.

By a simple application of the min-max principle for the self-adjoint operators, we have the following useful result.

Lemma 5 (Cf. [16]). In the general setting of Definition 4, the following holds true:

(A) If $H(q)$ is a diagonally monotone operator family in $\mathscr{H}$ with compact resolvent, then all eigenvalues $E_{i}^{H(q)}$ of $H(q)$ are also diagonally monotone.

(B) If $H(q)$ is diagonally monotone and $K$ is another selfadjoint operator in $\mathscr{H}$ independent of $q$, then $H(q)+K$ is also diagonally monotone.

Corollary 6 (Cf. $[17,18])$. Consider a parametric family of Schrödinger operators $H_{\Lambda}(q)=-\Delta_{\Lambda}+V(x, q)$ in a cube $\Lambda \subset \mathbb{R}^{d}, d \geq 1$, with a lower-bounded potential $V: \Lambda \times$ $\mathbb{R}^{J} \rightarrow\left[E_{*},+\infty\right)$ depending upon a vector-valued parameter $q \in \mathbb{R}^{J}, 1 \leq|J|<+\infty$. Let a probability measure $\mu$ on $\mathbb{R}$ with continuity modulus $\mathfrak{S}_{\mu}$ be given, and introduce the product measure $\mu^{\otimes J}$ on $\mathbb{R}^{J}$ and the corresponding probability measure induced on the operator families $V(\cdot, q)$ and $H(q)$. If the operator family $V(\cdot, q)$ is diagonally monotone, then for any interval $[a, b] \subset \mathbb{R}$ and any $E \in[a, b]$ one has

$$
\mathbb{P}\{\operatorname{dist}(\Sigma(H(q)), E) \leq \epsilon\} \leq C\left(E_{*}, b\right)|J||\Lambda| \mathfrak{S}_{\mu}(\epsilon) .
$$

\subsection{Stable Eigenvalue Concentration Estimate}

Definition 7. Let an integer $k \geq 0$ and a cube $B=B_{L_{k}}(u)$ be given. A sample $\omega$ is called 
(i) $(E, \epsilon)-\mathrm{NR}$ (nonresonant) in $B$ iff

$$
\operatorname{dist}\left(\Sigma\left(H_{B}(\omega)\right), E\right) \geq \epsilon
$$

(ii) $(E, \epsilon)$-CNR (completely nonresonant) in $B$ iff for all $j<L_{k+1} / L_{k}$

$$
\operatorname{dist}\left(\Sigma\left(H_{B_{j L_{k}}(u)}(\omega)\right), E\right) \geq \epsilon .
$$

Until Section 3 (where we formulate an Initial Length Scale estimate, Lemma 19), the positions of the sublattice sites $a$ with $\vartheta_{a}=1$ (impurities) will be considered fixed, so we do not rely on the substitution disorder due to the sample $\left\{\vartheta_{a}, a \in \mathscr{Z}^{d_{0}}\right\}$ and work with the displacements sample $\left\{\omega_{a}, a \in \mathscr{Z}\right\}$, without repeating it every time again, and often drop the $\vartheta$-dependence from notation.

Lemma 8. Fix any $k \geq 0$ and a cube $B=B_{L_{k}}(u) \subset \mathbb{R}^{d}$. Fix $\tau>1$. Then for any $\epsilon \geq L_{k}^{-(A-d) \tau}$ one has

$$
\mathbb{P}\{\omega \text { is not }(E, \epsilon)-C N R \text { in } B\} \leq C g^{-1} L_{k}^{d+1} \epsilon \text {. }
$$

Definition 9. Let a cube $B=B_{L_{k}}(u)$ and a real number $\tau>1$ be given. Denote $\bar{B}=B_{L^{\tau}}(u)$. The sample $\omega_{\bar{B}}$ is called

(i) $(E, \epsilon)$-SNR (strongly NR) in B iff for any complementary subsample $\omega_{\bar{B}}^{\perp}$ the full sample $\omega=\left(\omega_{\bar{B}}, \omega_{\bar{B}}^{\perp}\right)$ is $(E, \epsilon)$-NR in $B$,

(ii) $(E, \epsilon)$-SCNR (strongly CNR) in $B$ iff for any complementary subsample $\omega_{\bar{B}}^{\perp}$ the full sample $\omega=\left(\omega_{\bar{B}}, \omega_{\bar{B}}^{\perp}\right)$ is $(E, \epsilon)$-CNR in $B$.

A suitable value of $\tau>1$ will be fixed later, in Section 4, and the notations $B \equiv B_{L_{k}}(u), \bar{B} \equiv B_{L^{\tau}}(u), \omega_{\bar{B}}, \omega_{\bar{B}}^{\perp}$ will appear in a number of formulae and statements below. Usually the values of $k, L_{k} \in \mathbb{N}, u \in \mathscr{Z}$, and $\tau>1$ will be clear from the context.

In earlier works by Kirsch et al. [19] and by Boutet de Monvel and Stollmann [20], some functional-analytical facts pertaining to random alloy models in general and to surface models in particular have been established. Mutatis mutandis, some relevant definitions, arguments, and results from [19, 20] will be used below in the context of the surface model with random displacements disorder.

In Proposition 10, one refers to a certain subset $W \subset$ $\mathbb{R}^{d}$, which is formed in our case by the "wells" around the impurities, so $V(x ; \omega, \vartheta)<E^{(-)}$for $x \in W$ (cf. (3)), and $P$ is the exterior of the balls $B_{1 / 2}(a)$ around the impurities $a$ (i.e., with $\left.\vartheta_{a}=1\right)$. Given a cube $B \subset \mathbb{R}^{d}$, denote by $\widetilde{B}_{0}$ the section $B \cap \mathbb{R}^{d_{0}}$ by the impurity layer, and consider the finite set of scatterer indices

$$
\mathscr{A}_{B}=\left\{a^{\prime}=a+\mathbf{e}_{d_{0}+1} \text {, with } a \in \widetilde{B}_{0}\right\} .
$$

With this choice of $\mathscr{A}_{B}$, for each $a \in \widetilde{B}_{0}$ there exists a site $a^{\prime}$ outside the subset $W$ but at a uniformly bounded distance from $a \in \widetilde{B}_{0}$. Then we can write $H_{B}=\widetilde{H}_{B}+\widetilde{V}$, where $\widetilde{H}_{B}=$ $-\Delta+\widetilde{U}$ with

$$
\begin{aligned}
& \widetilde{U}(x)=\sum_{a \notin \mathscr{A}_{B}} \mathfrak{t}_{\vartheta_{a}}\left(x-X_{a}\right), \\
& \widetilde{V}(x)=\sum_{a \in \mathscr{A}_{B}} \mathfrak{t}_{\vartheta_{a}}\left(x-X_{a}\right) .
\end{aligned}
$$

Proposition 10 (cf. [20, Lemma 2.1]). Let $B \subset \mathbb{R}^{d}$ be an open cube, $H$ be a Schrödinger operator in $B$ with Dirichlet boundary conditions on the border of $B$, and $P \subset W$ be such that $\operatorname{dist}\left(P, W^{c}\right):=\kappa>0$. Then there exists $C=C(\kappa)$ such that the following holds.

Let $V_{0}$ and $V$ be uniformly locally summable in power $p$ (in $\left.L^{p}\right)$ with $p=2$ if $d \leq 3$ and $p>d / 2$ if $d>3$. Denote $H_{0}=$ $-\Delta+V_{0}, G_{0}(\lambda)=\left(H_{0}-\lambda\right)^{-1}$, and $H=-\Delta+V, G(\lambda)=(H-$ $\lambda)^{-1}$. Assume that $\Phi$ is an eigenfunction of $H$ with eigenvalue $\mu \in \varrho\left(H_{0}\right)$. Then

$$
\left\|\chi_{W} \Phi\right\| \geq \frac{\|\Phi\|}{1+C\left(\left\|G_{0}(\lambda)\right\|+\left\|G_{0}(\lambda) \nabla\right\|\right)} .
$$

Further, combining Proposition 11 and the Combes-Thomas estimate [21] for the under-the-barrier decay of the Green functions, one comes to the following.

Proposition 11 (cf. [19, Prop. 2.1], [20, Prop. 2.2]). Under the assumptions and with notations of Proposition 10, there exist $E_{1}>E_{0}$ and $C>0$ such that for every cube $B=B_{L}(x)$, every $\omega \in \Omega$, and every eigenfunction $\Phi$ of $H_{B}(\omega)$ with eigenvalue $E \in\left[E_{0}, E_{1}\right]$ we get

$$
\left\|\chi_{W} \Phi\right\| \geq C\|\Phi\|
$$

Proposition 12 (cf. [20, Prop. 2.3]).

$$
\mathbb{P}\left\{\Sigma\left(H_{B}\right) \cap J_{\epsilon} \neq \varnothing\right\} \leq C|B|^{2}\left|J_{\epsilon}\right| \text {. }
$$

Proof. Let $E_{n}=E_{n}\left(H_{B}\right)$ be the eigenvalues of $H_{B}$ numerated in the increasing order, counting multiplicity. We are mainly concerned with $E_{n} \in \widehat{I}$ (in the impurity band), which has two important consequences.

(i) Firstly, due to boundedness of the random potential, the operator $H_{B}(\omega)$ is a finite norm perturbation of the Laplacian $-\Delta_{B}$, and for the eigenvalues of the latter one has the well-known Weyl asymptotic formula [22]; see, for example, the discussion in [4, Section 4.1.8] in the context of the Wegner estimates. Specifically, it follows from [4, Theorem 4.1.25 and Corollary 4.1.26] that the number of eigenvalues of $H_{B}$ in $\widehat{I}$ admits uniform, nonrandom upper bounds by $O(|B|)$.

(ii) Secondly, inequality (20) shows that for such $E_{n}$ most of the "mass" of the respective eigenfunction $\Phi_{n}$ is in the subset $W$. Therefore, $E_{n}$ is monotone increasing in each parameter $q_{a}$, since increasing $q_{a}$ decreases the distance between the ath scatterer and any point $x \in W$ (in the "surface" layer), hence increasing the potential induced in $W$. This is the main reason why we consider in this paper a surface random displacements model as opposed to a "bulk" 
RDM. From this point on, we recover a complete analogy with the conventional surface alloy disorder studied in [20] (and not displacements disorder). As a result, only a few technical adaptations of the well-understood techniques from $[19,20]$ are required; we describe these adaptations below.

Specifically, notice that $E_{n}(q+t \mathbf{e})$ is continuously differentiable, with the derivative which can be calculated with the help of the first-order perturbation formula:

$$
\left.\frac{d}{d t}\right|_{t=s} E_{n}\left(\widetilde{H}_{B}+t \widetilde{V}\right)=\left(\widetilde{V} \Phi_{s}, \Phi_{s}\right) \geq C>0
$$

(we used again (20)). We conclude that $\widetilde{V}$ (and therefore $\widetilde{H}_{B}+$ $t \widetilde{V}$, too) is diagonally monotone. Now the claim follows from the Stollmann Lemma 3.

\section{Theorem 13.}

$$
\mathbb{P}\left\{\omega_{\bar{B}} \text { is not }(E, \epsilon)-C N R \text { in } B\right\} \leq C g^{-1} L^{2 d+1} \epsilon .
$$

Proof. The $(E, \epsilon)$-CNR property is violated in a cube $B=$ $B_{L_{k+1}}(u)$ if one of the smaller concentric cubes $B_{L}(u)$ with $L \in \llbracket L_{k}, L_{k+1} \rrbracket$ is not $(E, \epsilon)-\mathrm{NR}$, so the claim follows from Proposition 12 by counting the number of elements $L \in$ $\llbracket L_{k}, L_{k+1} \rrbracket$.

\section{Theorem 14.}

$$
\mathbb{P}\left\{\omega_{\bar{B}} \text { is not }(E, \epsilon)-S C N R \text { in } B\right\} \leq C g^{-1} L^{2 d+1} \epsilon .
$$

Proof. Let $\omega=\left(\omega_{\bar{B}^{\prime}}, \omega_{\bar{B}^{\prime}}^{\perp}\right)$. According to Definition 9, if $\omega_{\bar{B}}$ is $\operatorname{not}(E, \epsilon)$-SCNR, then for some integer $L \in \llbracket L_{k}, L_{k+1} \rrbracket \omega_{\bar{B}}$ is $\operatorname{not}(E, \epsilon)$-SNR in $B^{\prime}:=B_{L}(u)$. In other words, there exists a complementary subsample $\omega_{\bar{B}}^{\perp}$ such that the full sample $\omega=$ $\left(\omega_{\bar{B}}, \omega_{\bar{B}}^{\perp}\right)$ is $(E, \epsilon)$-resonant in $B^{\prime}$. Fix any $L \in \llbracket L_{k}, L_{k+1} \rrbracket$ and the corresponding cube $B^{\prime}$.

Next, consider any configuration $\omega_{\bar{B}}^{\perp}$ and take its zeroextension $\left(0_{\bar{B}}, \omega_{\bar{B}}^{\perp}\right)$ to the entire space; in other words, we consider $\omega_{\bar{B}}^{\perp}$ as a sample on its own but do not put any scatterers inside $\bar{B}$. We obtain by a simple calculation the following:

$$
\left\|\mathbf{1}_{B^{\prime}} g V\left(0, \omega_{\bar{B}^{\prime}}^{\perp}\right)\right\| \leq \text { Const } g L^{-(A-d) \tau} .
$$

Further, take any subsample $\omega_{\bar{B}}$, and identify it with its own zero-extension $\left(\omega_{\bar{B}}, 0_{\bar{B}} c\right)$ to the entire space, and let $\lambda_{i}\left(\omega_{\bar{B}}\right)$ be the eigenvalues of $H_{B}\left(\omega_{\bar{B}}\right)$. (One could take any complementary subsample $\omega_{\bar{B}}^{\perp}$; we take the zero-extension.) Then the eigenvalues $E_{i}(\omega)$ of $H_{B}(\omega)$, with any $\omega=\omega_{\bar{B}} \sqcup \omega_{\Lambda}^{\perp}$, obey

$$
\left|E_{i}(\omega)-\lambda_{i}\left(\omega_{\bar{B}}\right)\right| \leq g C L^{-(A-d) \tau} \leq \epsilon .
$$

Thus

$$
\begin{aligned}
& \mathbb{P}\left\{\omega_{\bar{B}}: \exists \omega_{\bar{B}}^{\perp}\left|E_{i}(\omega)-E\right| \leq \epsilon\right\} \\
& \quad \leq \mathbb{P}\left\{\omega_{\bar{B}}:\left|\lambda_{i}\left(\omega_{\bar{B}}\right)-E\right| \leq 2 \epsilon\right\} .
\end{aligned}
$$

Now the claim stems from Theorem 13.

\subsection{Eigenvalue Comparison Estimate}

Theorem 15. Let two sublattice sites $a, b \in \mathscr{Z}^{d_{0}}$ with $|a-b|>$ $2 L^{\tau}$ be given, and denote $B^{a}=B_{L}(a), B^{b}=B_{L}(b)$. Then for any $\epsilon>0$

$$
\mathbb{P}\left\{\operatorname{dist}\left(\Sigma\left(H_{B^{a}}(\omega)\right), \Sigma\left(H_{B^{b}}(\omega)\right)\right) \leq \epsilon\right\} \leq C L^{A+1+2 d} \epsilon
$$

The estimate given by Theorem 15 is not as accurate as its counterpart for a single cube (Theorem 13), and the proof is much less involved, but it is worth noticing that its role in the localization analysis is quite different, too. Optimality of the eigenvalue concentration (EVC) bound from Theorem 13 is the key to the proof of the exponential decay of the Green functions at fixed energy under the sharp condition $A>d$. Physically speaking, this gives rise to the absence of diffusion (see the discussion in the seminal paper by Fröhlich and Spencer [11]). On the mathematical level, Martinelli and Scoppola [23] showed, with the help of a nice application of Chebyshev's inequality in the extended disorder-energy space, that a fast decay of Green's functions implies absence of the absolutely continuous spectrum in the localization zone. However, the analysis of decay of the eigenfunctions and of their correlators requires a spectral reduction, or derivation of energy-interval (a.k.a. variable energy) MSA estimates, and the latter requires eigenvalue comparison analysis for distant cubes. For that purpose alone, an eigenvalue comparison estimate need not actually be very accurate, which is not surprising, but what comes as a rather unexpected bonus is that the comparison estimate allows one to significantly boost the results of the first scaling analysis in a "soft" way, as we shall see in Section 5.

Theorem 15 will not be used in Sections 3 and 4 .

Proof of Theorem 15. Denote $B^{a}=B_{L}(a)$ and $B^{b}=B_{L}(b)$. Consider an eigenvalue $E_{i}^{a}(\omega)$ of $H_{B^{a}}(\omega)$ and $E_{j}^{b}(\omega)$ of $H_{B^{b}}(\omega)$. Condition on all random amplitudes $\left\{\omega_{c}, c \in \mathbb{Z}^{d} \backslash \omega_{a}\right\}$ and identify $\omega_{a}$ with a real parameter $s$. Then $H_{B^{a}}$ as well as $H_{B^{b}}$ and their eigenvalues $E_{i}^{a}, E_{j}^{b}$ depend upon a single parameter $s \equiv \omega_{a}$. Our goal is to compare their sensitivity to the variations of $s$. To this end, consider the smooth operator family $H_{B_{a}}(s)=\widetilde{H}_{B_{a}}+s U_{a}(s)$, where

$$
\begin{aligned}
\widetilde{H}_{B^{a}} & =-h^{2} \Delta+\sum_{c \neq a} \omega_{c} \mathfrak{u}(\cdot-c)+s U_{a}(s), \\
U_{a}(x, s) & =\mathfrak{u}(x-a) \mathbf{1}_{B^{a}}(x) .
\end{aligned}
$$

Similarly define

$$
\begin{aligned}
H_{B^{b}}(s) & =\widetilde{H}_{B_{b}}+s U_{b}(s), \\
U_{b}(x, s) & =\mathfrak{u}(x-a) \mathbf{1}_{B_{b}}(x) .
\end{aligned}
$$

Then

$$
\max _{z \in B_{b}} U_{b}(z) \leq(\widetilde{M} L)^{-A}<C(d) L^{-A} \leq \min _{z \in B_{a}} U_{a}(z) .
$$


Therefore,

$$
\begin{aligned}
& U_{a} \mathbf{1}_{B_{a}} \geq\left(C_{d} L\right)^{-A} \mathbf{1}_{B_{a}}, \\
& U_{b} \mathbf{1}_{B_{a}} \leq(\widetilde{M} L)^{-A} \mathbf{1}_{B_{b}} .
\end{aligned}
$$

Furthermore, computing the gradient of the potential $x \mapsto$ $|x|^{-A}$, for $\widetilde{M}$ large enough we get that

$$
\begin{aligned}
\frac{\mathrm{d}}{\mathrm{d} s}\left(E_{i}^{\prime}(s)-E_{j}^{\prime \prime}(s)\right) & \geq\left(\widetilde{\mathrm{M}}^{-A-1}-C_{d}^{-A-1}\right) L^{-A-1} \\
& \geq \frac{\widetilde{\mathrm{M}}^{-A-1}}{2} L^{-A-1}>0 .
\end{aligned}
$$

The probability measure of the random variable $E_{i}^{a}\left(\omega_{a}\right)-$ $E_{j}^{b}\left(\omega_{a}\right)$ is therefore the image of $\mu_{V}$ (the measure of $\omega_{a}$ ) by the strictly monotone mapping $s \mapsto\left(E_{i}^{a}(s)-E_{j}^{b}(s)\right)$, with the derivative lower-bounded by $C L^{-A-1}>0$, so the Lipschitz continuity of $\mu_{V}$ is preserved up to a factor $C^{-1} L^{A+1}$. Now the claim follows by counting the number of eigenvalues in $I$, as in Proposition 12.

\section{Impurity Band: Location and Spectral Gap}

An upper bound on the ground state energy $E_{*}$ can be obtained by the variational principle.

\section{Multiscale Analysis}

Given $\alpha>1$ and $1<L_{0} \in \mathbb{N}$, define recursively an integer sequence $L_{k}$

$$
L_{k}:=\left\lfloor L_{k-1}^{\alpha}\right\rfloor, \quad k \geq 1 .
$$

Further, let $m_{0}>0$, and set for $k \geq 0$

$$
\begin{gathered}
m_{k}:=m_{0} \prod_{j=0}^{k}\left(1-\eta_{j}\right), \\
\eta_{k}:=2 L_{k}^{-c \alpha}, \quad c>0,
\end{gathered}
$$

with $c>0$ to be specified in the proof of Corollary 21. Clearly, with $L_{0}$ large enough we have a convergent product $\prod_{j \geq 0}(1-$ $\left.\eta_{j}\right) \geq 1 / 2$, so $m_{k} \geq m_{0} / 2$.

Given $A>2 d$ and $\tau>1$, we define two positive sequences

$$
\begin{aligned}
\delta_{k} & :=e^{-m_{k} L_{k}}, \\
\epsilon_{k} & :=L_{k}^{-(A-d) \tau} .
\end{aligned}
$$

Definition 16. Let an integer $k \geq 0$ and a cube $B=B_{L_{k}}(u)$ be given. Denote $\partial B_{L_{k}}(u):=B_{L_{k}}(u) \backslash B_{L_{k}-2}(u)$. A sample $\omega$ is called

(1) $(E, \delta)$-NS (nonsingular) in $B$ iff $E \notin \Sigma\left(H_{B}(\omega)\right)$ and

$$
\left\|\mathbf{1}_{\partial B_{L_{k}}} G_{B_{L_{k}}}(E, \omega) \mathbf{1}_{B_{L_{k} / 3}}\right\| \leq\left(3 L_{k}\right)^{-d} \delta .
$$

Definition 17. Let $\tau>1$ and a cube $B=B_{L_{k}}(u), k \geq 0$ be given. Denote $\bar{B}=B_{(1 / 2) L_{k}^{\tau}}(u)$ and introduce the decomposition $\omega=$ $\left(\omega_{\bar{B}}, \omega_{\bar{B}}^{\perp}\right)$. Let $\mathscr{P}$ be one of the properties $(E, \delta)$-NS, $(E, \epsilon)$-NR, or $(E, \epsilon)$-CNR relative to $B$. We will say that a subsample $\omega_{\bar{B}}$ has a strong (or stable) property $\mathscr{P}$ iff for any complementary subsample $\omega_{\bar{B}}^{\perp}$ the full sample $\omega=\left(\omega_{\bar{B}}, \omega_{\bar{B}}^{\perp}\right)$ has property $\mathscr{P}$ in $B$. Respectively, the three aforementioned notations are replaced by $(E, \delta)$-SNS, $(E, \epsilon)$-SNR, and $(E, \epsilon)$-SCNR.

Note that an event $\left\{\omega_{\bar{B}}\right.$ has strong property $\left.\mathscr{P}\right\}$ is measurable with respect to the $\sigma$-algebra $\mathfrak{F}_{\bar{B}}$ generated by $\left\{X_{a}, a \in\right.$ $\bar{B}\}$; hence any collection of such events relative to disjoint cubes $B_{L}\left(u_{i}\right), i \in \llbracket 1, M \rrbracket$ is independent.

Definition 18. (i) A cube $B_{L_{k+1}}(u)$ is called $\left(E, \delta_{k}, \tau, K\right)$-good iff it contains no collection of $K$ or more cubes $\left\{B_{L_{k}}\left(x_{i}\right), 1 \leq\right.$ $\left.i \leq S_{k+1}\right\}$ with pairwise $L_{k}^{\tau}$-distant centers, neither of which is $\left(E, \delta_{k}\right)$-NS.

(ii) The cube $B_{L_{k+1}}(u)$ is called $\left(E, \delta_{k}, \tau, K\right)$-strongly good $\left(\left(E, \delta_{k}, \tau, K\right)\right.$-S-good) iff it contains no collection of $K$ cubes $\left\{B_{L_{k}}\left(x_{i}\right), 1 \leq i \leq K\right\}$, with pairwise $L_{k}^{\tau}$-distant centers, neither of which is $\left(E, \delta_{k}\right)$-SNS.

Lemma 19 (ILS estimate). Let $\tau>1$. There exist $L_{*} \in \mathbb{N}$ and an interval $I=[-3 \mathscr{E},-2 \mathscr{E}]$ with $\mathscr{E}>0$, such that for any $L_{0} \geq L_{*}, m_{0}>0, \mathfrak{g}>0$ and the impurity concentration $\rho>0$ small enough, one has, with $\delta_{0}=e^{m_{0} L_{0}}$,

$$
\sup _{E \in I} \mathbb{P}\left\{B_{x} \text { is not }\left(E, \delta_{0}, \tau\right)-S N S\right\} \leq L_{0}^{-\mathfrak{s}} .
$$

Proof. Fix $L_{0} \in \mathbb{N}$; consider a cube $B=B_{L_{0}}(u)$ and the local Hamiltonian $H_{B}(\omega)$. Observe that the condition

$$
\theta_{a}=0 \quad \forall a \in B_{L_{0}+r_{0}}
$$

(absence of impurity atoms in the augmented cube $B_{L_{0}+r_{0}}(u)$ ) implies that

$$
\inf _{x \in B} V(x, \omega, \vartheta) \geq-\mathscr{E}
$$

thus

$$
\operatorname{dist}\left[E, \Sigma\left(H_{B}(\omega, \vartheta)\right), E\right] \geq \mathscr{E} \quad \forall E \in[-3 \mathscr{E},-2 \mathscr{E}] .
$$

By the Combes-Thomas estimate [21], we then have for any $E \in I \equiv[-3 \mathscr{E},-2 \mathscr{E}]$

$$
\left\|\mathbf{1}_{\partial B} G_{B}(E) \mathbf{1}_{B_{(1 / 3) L_{k}}(u)}\right\| \leq C \mathscr{E}^{-1} e^{-c \mathscr{E} L_{k}},
$$

for some $C, c \in(0,+\infty)$. With an appropriate choice of $L_{*}$, this implies the $\left(E, \delta_{0}\right)$-nonsingularity of $B_{L_{0}}(u)$, where $\delta_{0}=$ $e^{-m_{0} L_{0}}$ and, for example, $m_{0}=(1 / 2) c \mathscr{C}>0$.

Further,

$$
\mathbb{P}\left\{\exists a \in B_{L_{0}+r_{0}}(u): \theta_{a} \neq 0\right\} \leq\left|B_{L_{0}+r_{0}}(u)\right| \rho,
$$

and the RHS can be made arbitrarily small by taking the concentration of impurities $\rho$ small enough. In particular, it can be made smaller than $L_{0}^{-\mathcal{3}}$, once $L_{0}$ is fixed. 
The next statement is a standard result of the MSA, essentially going back to [12, Lemma 4.2] and streamlined in [24, Section 5] and later in [25, Lemma 2].

Lemma 20 (conditions for nonsingularity). Consider a cube $B=B_{L_{k+1}}(u)$ and suppose that

(i) $B$ is $\left(E, \epsilon_{k+1}\right)-N R$ with $\epsilon_{k+1} \geq \delta_{k}^{1-c}$, for some $\epsilon_{k+1}, \delta_{k}, c \in$ $(0,1)$;

(ii) $B$ is $\left(E, \delta_{k}, \tau, K\right)$-good, with $K \geq 0$ such that

$$
N:=\left\lfloor\frac{L_{k+1}}{L_{k}}\right\rfloor-10 K\left\lfloor L_{k}^{\tau}\right\rfloor \geq 1 .
$$

Then $B$ is $\left(\delta_{k}^{N+c}, E\right)-N S$.

In our case $\delta_{k}=e^{-m_{k} L_{k}}$ and $\epsilon_{k+1}=e^{-O\left(\ln L_{k+1}\right)}$; hence the condition $\epsilon_{k+1} \geq \delta_{k}^{1-c}$ in hypothesis (ii) is fulfilled for any $c \in(0,1)$ and large $L_{0}$.

Corollary 21 (conditions for strong nonsingularity). Let a cube $B=B_{L_{k+1}}(u), k \geq 0$, be given and suppose that

(i) $B$ is $\left(E, \epsilon_{k+1}\right)-S N R$;

(ii) $B$ is $\left(E, \delta_{k}, \tau, K\right)$-S-good, with $K \geq 0$ such that (44) holds.

Then $B$ is $\left(E, \delta_{k+1}\right)$-SNS.

Proof. Denote $\bar{B}=B_{L_{k+1}}^{\tau}(u)$. One has to show that, with a fixed sample $\omega_{\bar{B}}^{\perp}$ satisfying the hypotheses (i)-(ii), the cube $B$ is $\left(E, \delta_{k+1}\right)$-NS for the sample $\left(\omega_{\bar{B}}, \omega_{\bar{B}}^{\perp}\right)$ regardless of the complementary sample $\omega_{\bar{B}}^{\perp}$.

First, notice that the condition (i) is already stable with respect to $\omega_{\bar{B}}^{\perp}$.

Next, by (ii) there exist at most $K-1$ cubes $B_{(1 / 2) L_{k}^{\tau}}\left(x_{i}\right)$ such that any ball $B_{L_{k}}(x)$ with $x \notin \bigcup_{i=1}^{K} B_{L_{k}^{\tau}}\left(x_{i}\right)$ is $\left(E, \delta_{k}\right)$-SNS. The support of $\omega_{\bar{B}}^{\perp}$ lies outside all the cubes $B_{(1 / 2) L_{k}^{\tau}}\left(x_{i}\right)$; hence the distant sample $\omega_{\bar{B}}^{\perp}$ cannot affect the strong nonsingularity property of the cubes $B_{(1 / 2) L_{k}^{\tau}}\left(x_{i}\right)$. Applying Lemma 20, we see that the cube $B$ is $\left(E, \widetilde{\delta}_{k+1}\right)$-NS with

$$
\begin{aligned}
-\ln \widetilde{\delta}_{k+1}= & N_{k+1} m_{k} L_{k}-C^{\prime} \ln L_{k+1} \\
& +\left(\ln \left(3 L_{k+1}\right)^{d}-\ln \left(3 L_{k+1}\right)^{d}\right) \\
\geq & L_{k} Y_{k+1} m_{k}\left(1-\frac{10 K L_{k}^{\tau}}{L_{k}^{\alpha}}-\frac{C^{\prime \prime} \ln L_{k+1}}{m_{k} L_{k+1}}\right) \\
& +\ln \left(3 L_{k+1}\right)^{d} \\
\geq & L_{k+1} m_{k}\left(1-\eta_{k}\right)+\ln \left(3 L_{k+1}\right)^{d},
\end{aligned}
$$

where $\eta_{k}$ is as in (35), with $c=\alpha-\tau>0$. Thus $B$ is $\left(E, \delta_{k+1}\right)$ SNS.

Until the end of this section, we will need to examine only the nonsingularity properties of the cubes having nonempty intersection with the sublattice $\mathscr{Z}^{d_{0}}$ (the impurity layer), since we are concerned only with energies $E \in \widehat{I}$, and any cube outside this layer has energies above $\widehat{I}$. Moreover, since we are free to choose on any scale the partition of $\mathbb{R}^{d}$ into a union of cubes of size $L_{k}$, we cover first $\mathscr{Z}^{d_{0}}$ by cubes $B_{L_{k}}(a)$ with $a \in \mathscr{Z}^{d_{0}}$ and then decompose the rest of the space into $L_{k}$-cubes having empty intersection with $\mathscr{Z}^{d_{0}}$. We will not repeat this fact every time again.

Lemma 22. Let two real numbers $A>d$ and $\tau>1$ be given. Consider a cube $B_{L_{k+1}}(x)$ and let $\epsilon_{k+1}=L_{k+1}^{-(A-d) \tau}$. Then

$$
\begin{aligned}
& \mathbb{P}\left\{\omega_{\bar{B}}: \exists \omega_{\bar{B}}^{\perp} B_{L_{k+1}}(x) \text { is not }\left(E, \epsilon_{k+1}\right)-S C N R\right\} \\
& \quad \leq \frac{1}{2} L_{k+1}^{-(A-d) \tau+2 d+1} .
\end{aligned}
$$

Proof. By Definition 17, if $B_{L_{k+1}}(z)$, with $z \in\{x, y\}$, is not $\left(E, \epsilon_{k+1}\right)$-SCNR for some fixed $E$, then for some $R \in$ $\llbracket L_{k}, L_{k+1} \rrbracket$ the cube $B_{R}(x)$ is not $\left(E, \epsilon_{k+1}\right)$-SNR. Even the largest among them, $B_{L_{k+1}}(z)$, is surrounded by a belt of width $L_{k+1}^{\tau}$. By Theorem 14,

$$
\mathbb{P}\left\{\omega_{\bar{B}}: \exists \omega_{\bar{B}}^{\perp} \operatorname{dist}\left[\Sigma\left(H_{B_{R}(z)}\right), E\right] \leq 2 \epsilon_{k+1}\right\} \lesssim L^{2 d+1} \epsilon_{k+1},
$$

so the claim follows by counting the number of values $R \in$ $\llbracket L_{k}, L_{k+1} \rrbracket$.

Lemma 23. Let $A=3 d+4 \gamma$ with $\gamma>0$ and $\tau>(2 d+1) / 2 \gamma$ be given. Set

$$
\begin{aligned}
\varrho & =\frac{\gamma}{d+4 \gamma}, \\
\sigma & =\gamma d^{-1}, \\
\alpha & =(1+\sigma) \tau, \\
\mathfrak{S} & =(A-d) \tau-(2 d+1) ;
\end{aligned}
$$

then

$$
\mathfrak{g}-\alpha d>\gamma \tau>0
$$

Furthermore, assume that

$$
\mathbb{P}\left\{B_{L_{k}}(x) \text { is not }\left(E, \delta_{k}\right)-S N S\right\} \lesssim L_{k}^{-\mathfrak{3}}
$$

and let $K=2 M(1+\sigma) \mathfrak{g} / \gamma \leq L_{k}^{\alpha-\tau}$. Then for $L_{0}$ large enough

$$
\mathbb{P}\left\{B_{L_{k+1}}(u) \text { is not }\left(E, \delta_{k+1}, K\right)-S \text {-good }\right\} \leq \frac{1}{4} L_{k+1}^{-M \mathfrak{3}} .
$$

Proof. Equation (49) follows by a simple calculation:

$$
\begin{aligned}
\mathfrak{G}-\alpha d & =(A-d) \tau-(2 d+1)-(1+\sigma) \tau d \\
& =\tau[(d+3 \gamma)-(1+\sigma) d]-(2 d+1) \\
& =\tau[3 \gamma-\sigma d]-(2 d+1) \\
& \geq \tau[3 \gamma-\gamma]-(2 d+1)>\gamma \tau .
\end{aligned}
$$


Further, by Definition 17, an event

$$
\begin{gathered}
\mathscr{B}_{x}=\left\{B_{L_{k}}(x) \text { is }\left(E, \delta_{k}, K\right) \text {-S-bad or not }\left(E, \epsilon_{k+1}\right)\right. \\
\text {-SNR }\}
\end{gathered}
$$

is $\mathfrak{F}\left(B_{(1 / 2) L_{k}^{\tau}}(x)\right)$-measurable, and so for $K$ disjoint cubes $B_{(1 / 2) L_{k}^{\tau}}\left(x_{i}\right), 1 \leq i \leq K$, we obtain

$$
\mathbb{P}\left\{\bigcap_{i=1}^{K} \mathscr{B}_{x_{i}}\right\}=\prod_{i=1}^{K} \mathbb{P}\left\{\mathscr{B}_{x_{i}}\right\} \leq p_{k}^{K} .
$$

By (52), we have $(\mathfrak{G}-\alpha d) / \alpha=\gamma \tau /(1+\sigma) \tau=\gamma /(1+\sigma)$. Thus with $K=2 M \mathfrak{s}(1+\sigma) \gamma^{-1}$, one has for the maximal number $S(\omega)$ of pairwise $L_{k}^{\tau}$-distant singular cubes $B_{(1 / 2) L_{k}^{\tau}}\left(x_{i}\right)$ inside $B_{L_{k+1}}(u)$ :

$$
\mathbb{P}\{S(\omega) \geq K\} \leq C L_{k+1}^{K d} p_{k}^{K} \leq C L_{k+1}^{-K(\mathfrak{g} / \alpha-d)}<\frac{1}{4} L_{k+1}^{-M \mathfrak{I}} .
$$

This proves inequality (51).

For future use, note that one can take, for example,

$$
M=\frac{\gamma}{4(1+\sigma) \mathfrak{g}} L_{k}^{\alpha-\tau}>L_{k}^{\sigma \tau / 2}
$$

The last inequality holds for all $k$ with $L_{0}$ large enough.

Lemma 24 (scale induction). Assume that the bound

$$
\begin{aligned}
& \mathbb{P}\left\{\exists E \in \widehat{I} \exists z \in\{x, y\} B_{L_{j}}(z) \text { is not }\left(E, \delta_{j}\right) \text {-SNS }\right\} \\
& \quad \leq L_{j}^{-\mathfrak{s}}
\end{aligned}
$$

holds for $j=k \geq 0$. Then it also holds for $j=k+1$.

Proof. By Corollary 21, if the cube $B_{L_{k+1}}(z)$ is not $\left(E, \delta_{k+1}\right)$-SNS, then either

(i) $B_{L_{k+1}}(z)$ is not $\left(E, \epsilon_{k+1}\right)-\mathrm{SNR}$, or

(ii) $B_{L_{k+1}}(z)$ is not $\left(E, \delta_{k}, K\right)$-S-good.

By Lemmas 22 and 23, the probabilities of both events are upper-bounded by $(1 / 2) L_{k+1}^{-\mathfrak{a}}$, so the claim follows.

Since the starting point for the scale induction (the Initial Length Scale estimate) is provided by Lemma 19, by induction on $k$ we come to the conclusion of the multiscale analysis of our model.

Theorem 25. Consider the random Hamiltonian $H(\omega)=$ $-\Delta+V(x, \omega)$. Fix an interval $I \subset \mathbb{R}$. For any $A>2 d$ there exist $\mathfrak{G}_{*}=\mathfrak{G}_{*}(A)<+\infty$ and $L_{*} \in \mathbb{N}$ such that if $L_{0} \geq L_{*}$ and the inequality

$$
\sup _{E \in I} \mathbb{P}\left\{B_{L_{k}}(x) \text { is not }\left(E, \delta_{k}\right)-S N S\right\} \leq L_{k}^{-\mathfrak{Z}}
$$

holds for $k=0$ with $\mathfrak{g} \geq \mathfrak{G}_{*}$, then it also holds for all $k \geq 1$.

\section{Derivation of Strong Dynamical Localization}

\subsection{Enhancement of the MSA Estimates}

Theorem 26. Under the assumptions and with notations of Theorem 25, for any two cubes $B_{L_{k}}(x), B_{L_{k}}(y)$ with $|x-y| \geq L_{k}^{\tau}$ one has for some $\varrho>0$

$$
\mathbb{P}\{\exists E\} \leq L_{k}^{-L_{k}^{e}} .
$$

Proof. It suffices to modify the proof of Lemma 23 (and of the Lemma 24 stemming from it) in the following way. Instead of the condition that $B_{L_{k}}(z)$ be $\left(E, \epsilon_{k}\right)$-strongly nonresonant we employ the condition of $\left(E, \epsilon_{k}\right)$-nonresonance. As a result, the choice of $\epsilon_{k+1}$ is no longer restricted to $\epsilon_{k+1} \geq L_{k}^{-(A-d) \tau}$ : we can take an arbitrarily small value of $\epsilon_{k+1}$. Indeed, the strong nonresonance is required for the scale induction, but now we enhance the MSA estimates scale by scale, individually for each $L_{k}$, without resorting to another scale induction.

Consequently, we can also replace a fixed, scaleindependent parameter $K$ (figuring in the condition " $B_{L_{k+1}}(z)$ is $\left(E, \delta_{k+1}, K\right)$-good" by a growing value). Setting $K=K_{k+1}=(1 / 2) L_{k}^{1-\tau / \alpha}=(1 / 2) L_{k}^{1-\sigma / \alpha}$, the claim follows.

5.2. Strong Dynamical Localization. It is well-known by now that the energy-interval MSA estimates imply strong dynamical localization; the shortest derivation is due to Germinet and Klein [24] who operated with the eigenfunction correlators in the entire space. Their argument becomes particularly simple in the situation where one proves first the decay bound for the eigenfunction correlators in finite cubes, where it can be encapsulated in a fairly elementary functional-analytical lemma, as was shown in our prior papers, for example, [25, 26].

Proposition 27 (cf. [26, Thm. 7], [25, Thm. 3]). Suppose that a bound of the form

$$
\begin{aligned}
& \mathbb{P}\left\{\exists E \in \widehat{I} \exists z \in\{x, y\}: B_{L_{k}(z)} \text { is not }\left(E, \delta_{k}\right)-N S\right\} \\
& \quad \leq f\left(L_{k}\right)
\end{aligned}
$$

is established for some function $f \geq 0$ and all $k \geq 0$. Then for $|x-y|=: R \in\left[3 L_{k}, 3 L_{k+1}\right]$, one has

$$
\mathbb{E}\left[\chi_{x} \phi_{I}(H(\omega)) \chi_{y}\right] \leq C_{1} R^{d} f\left(R^{\alpha^{-1}}\right)+C_{2} e^{-m R} .
$$

Taking into account the MSA estimates from Theorem 26, assertion (B) of Theorem 1 follows directly from Proposition 27.

\section{Conflicts of Interest}

The author declares that there are no conflicts of interest.

\section{References}

[1] F. Klopp, "Localization for semiclassical continuous random Schrödinger operators. II. The random displacement model," 
Helvetica Physica Acta. Physica Theoretica. Societatis Physicae Helveticae Commentaria Publica, vol. 66, no. 7-8, pp. 810-841, 1993.

[2] W. Kirsch, "An invitation to random Schrödinger operators," in Panorama et Synthèses, vol. 25, pp. 1-119, 2008.

[3] I. M. Lifshitz, "Energy spectrum structure and quantum states of disordered condensed systems," Soviet Physics-Uspekhi, vol. 7, pp. 549-573, 1965.

[4] P. Stollmann, Caught by disorder, vol. 20 of Progress in Mathematical Physics, Birkhäuser Boston, Inc., Boston, MA, 2001.

[5] J. Lott and G. Stolz, "The spectral minimum for random displacement models," Journal of Computational and Applied Mathematics, vol. 148, no. 1, pp. 133-146, 2002.

[6] J. Baker, M. Loss, and G. Stolz, "Minimizing the ground state energy of an electron in a randomly deformed lattice," Communications in Mathematical Physics, vol. 283, no. 2, pp. 397-415, 2008.

[7] J. Baker, M. Loss, and G. Stolz, "Low energy properties of the random displacement model," Journal of Functional Analysis, vol. 256, no. 8, pp. 2725-2740, 2009.

[8] F. Klopp, M. Loss, S. Nakamura, and G. Stolz, "Understanding the random displacement model: From ground state properties to localization," Spectral Analysis of Quantum Hamiltonians: Spectral Days 2010, pp. 183-219, 2012.

[9] W. Kirsch, P. Stollmann, and G. Stolz, "Anderson localization for random Schrödinger operators with long range interactions," Communications in Mathematical Physics, vol. 195, no. 3, pp. 495-507, 1998.

[10] J. Fröhlich, F. Martinelli, E. Scoppola, and T. Spencer, "Constructive proof of localization in the Anderson tight binding model," Communications in Mathematical Physics, vol. 101, no. 1, pp. 21-46, 1985.

[11] J. Fröhlich and T. Spencer, "Absence of diffusion in the Anderson tight binding model for large disorder or low energy," Communications in Mathematical Physics, vol. 88, no. 2, pp. 151184, 1983.

[12] H. von Dreifus and A. Klein, "A new proof of localization in the Anderson tight binding model," Communications in Mathematical Physics, vol. 124, no. 2, pp. 285-299, 1989.

[13] J. Bourgain and C. E. Kenig, "On localization in the continuous Anderson-Bernoulli model in higher dimension," Inventiones Mathematicae, vol. 161, no. 2, pp. 389-426, 2005.

[14] F. Germinet and A. Klein, "A comprehensive proof of localization for continuous Anderson models with singular random potentials," Journal of the European Mathematical Society, vol. 15, no. 1, pp. 53-143, 2013.

[15] P. Stollmann, "Wegner estimates and localization for continuum Anderson models with some singular distributions," Archiv der Mathematik, vol. 75, no. 4, pp. 307-311, 2000.

[16] V. Chulaevsky, "A wegner-type estimate for correlated potentials," Mathematical Physics, Analysis and Geometry, vol. 11, no. 2, pp. 117-129, 2008.

[17] A. Boutet de Monvel, V. Chulaevsky, P. Stollmann, and Y. Suhov, "Wegner-type bounds for a multi-particle continuous Anderson model with an alloy-type external potential," Journal of Statistical Physics, vol. 138, no. 4-5, pp. 553-566, 2010.

[18] V. Chulaevsky, A. Boutet de Monvel, and Y. Suhov, "Dynamical localization for a multi-particle model with an alloy-type external random potential," Nonlinearity, vol. 24, no. 5, pp. 14511472, 2011.
[19] W. Kirsch, P. Stollmann, and G. Stolz, "Localization for random perturbations of periodic Schrödinger operators," Random Operators and Stochastic Equations, vol. 6, no. 3, pp. 241-268, 1998.

[20] A. Boutet de Monvel and P. Stollmann, "Dynamical localization for continuum random surface models," Archiv der Mathematik, vol. 80, no. 1, pp. 87-97, 2003.

[21] J. M. Combes and L. Thomas, "Asymptotic behaviour of eigenfunctions for multiparticle Schrödinger operators," Communications in Mathematical Physics, vol. 34, pp. 251-270, 1973.

[22] H. Weyl, "Das asymptotische Verteilungsgesetz der Eigenwerte linearer partieller Differentialgleichungen (mit einer Anwendung auf die Theorie der Hohlraumstrahlung)," Mathematische Annalen, vol. 71, no. 4, pp. 441-479, 1912.

[23] F. Martinelli and E. Scoppola, "Remark on the absence of absolutely continuous spectrum for -dimensional Schrödinger operators with random potential for large disorder or low energy," Communications in Mathematical Physics, vol. 97, no. 3, pp. 465-471, 1985.

[24] F. Germinet and A. Klein, "Bootstrap multiscale analysis and localization in random media," Communications in Mathematical Physics, vol. 222, no. 2, pp. 415-448, 2001.

[25] V. Chulaevsky, "Exponential scaling limit of the single-particle Anderson model via adaptive feedback scaling," Journal of Statistical Physics, vol. 162, no. 3, pp. 603-614, 2016.

[26] V. Chulaevsky, "From fixed-energy localization analysis to dynamical localization: an elementary path," Journal of Statistical Physics, vol. 154, no. 6, pp. 1391-1429, 2014. 


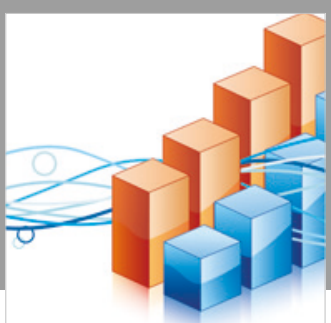

Advances in

Operations Research

\section{-n-m}
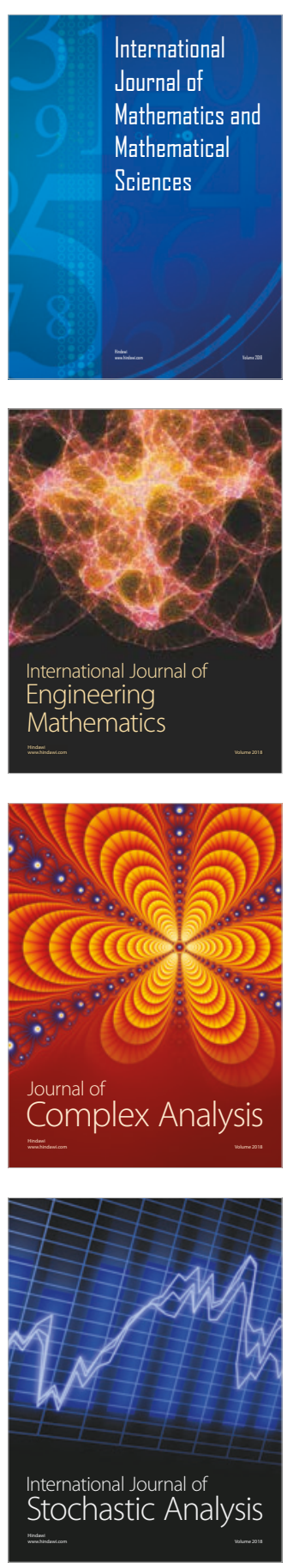
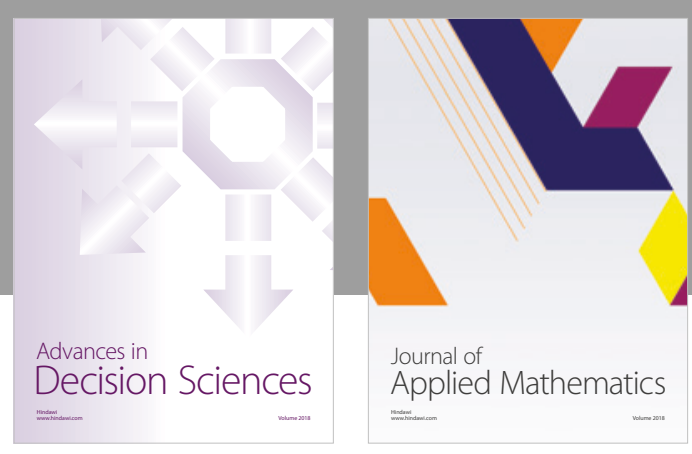

Journal of

Applied Mathematics
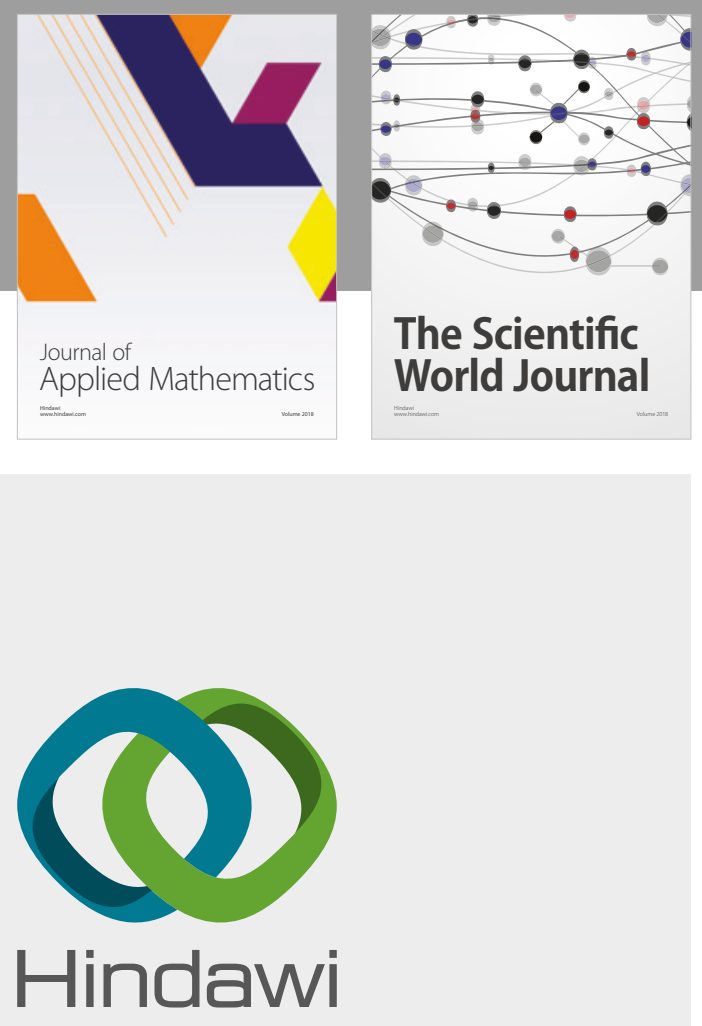

Submit your manuscripts at

www.hindawi.com

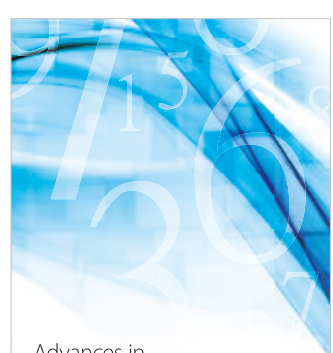

Advances in
Numerical Analysis
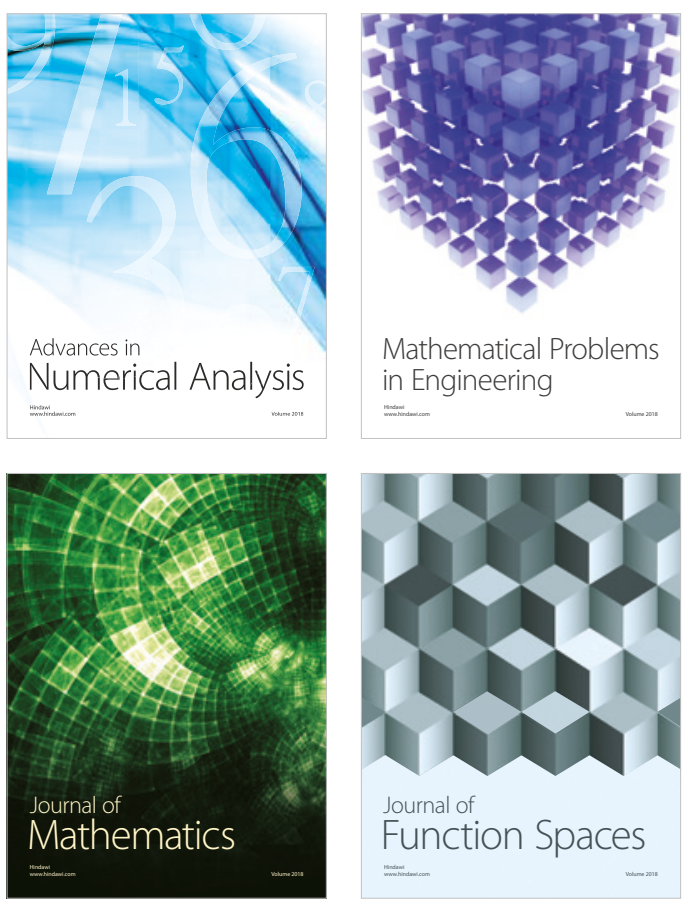

Mathematical Problems in Engineering

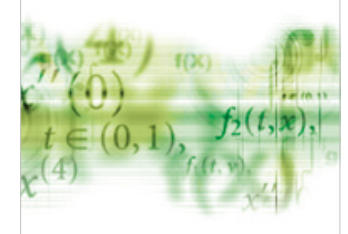

International Journal of

Differential Equations

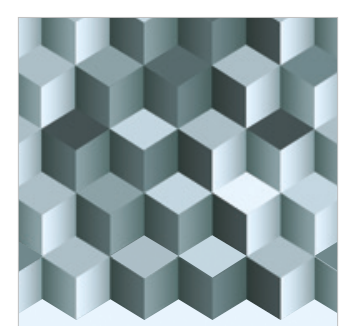

Journal of

Function Spaces

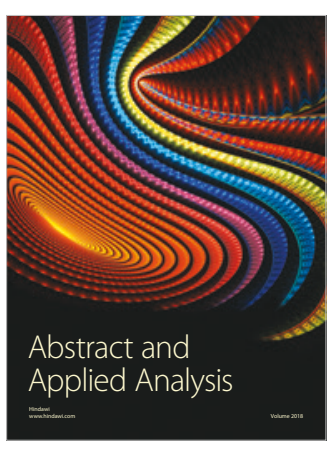

The Scientific

World Journal

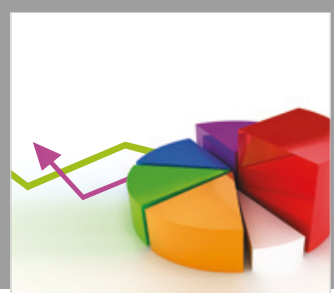

Journal of

Probability and Statistics
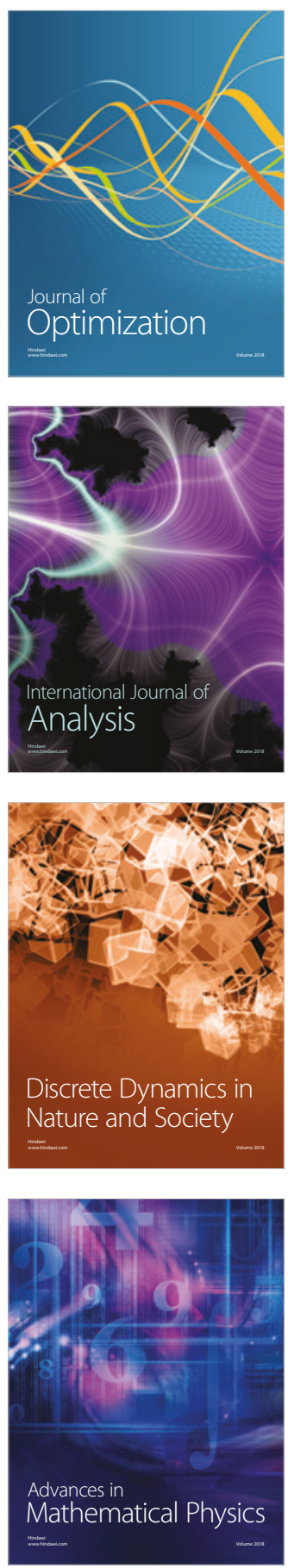